\title{
A RELAÇÃO ENTRE ESTRESSE OCUPACIONAL E JUSTIÇA ORGANIZACIONAL: UM ENSAIO
}

\author{
The Relationship Between Occupational Stress and Organizational Justice: An Essay \\ La Relation Entre Le Stress Professionnel Et La Justice Organisationnelle: Un Essai \\ La Relación Entre Estrés Ocupacional Y Justicia Organizacional: Un Ensayo
}

\begin{abstract}
Paulo Eduar do Benzoni ${ }^{1}$
Professor Titular, Pesquisadore Líder de Grupo de Pesquisas em Saúde Mental nos Contextos Institucionais na Universidade Paulista - UNIP/Campus Ribeirão Preto SP. Programa Individual de Pesquis as para Docentes da Universidade Paulista - UNIP

Juliana Gomes Ervolino Graduanda em Psicologia na UNIP - Universidade Paulista Campus Ribeirão Preto. Iniciação Científica Voluntária.

Michel da Matta Simões ${ }^{3}$ Graduando em Psicologia na Universidade Paulista - UNIP Campus Ribeirão Preto. Bolsista do Programa $\mathrm{PIBIC} / \mathrm{CNPq}$.
\end{abstract}

\begin{abstract}
Resumo
O estudo do estresse ocupacional, suas fontes, dinâmica de funcionamento e consequências tem sido largamente estudado. Há consenso de que o ambiente de trabalho consiste em uma poderosa fonte de estresse ocupacional. Tomando o construto justiça organizacional, aventa-se a hipótese de que um ambiente de trabalho marcado por injustiças organizacionais pode ser um poderoso estressor, uma vez que exige constantes adaptações do indivíduo para lidar com tais injustiças. Este trabalho, de cunho teórico, propõe uma reflexão da relação entre os construtos estresse ocupacional e justiça organizacional, concluindo a partir da literatura que um ambiente de trabalho marcado por injustiças pode sim ser um potencial estressor e aponta a importância desta relação ser investigada empiricamente.
\end{abstract}

Palavras Chave: Estresse; Justiça organizacional; Estressores.

\begin{abstract}
The study of the occupational stress, its sources, functioning dynamic and consequences has been largely studied. There is a consensus that the working environment consists in a powerful source of the occupational stress. Taking the organizational justice construct, it is considered the hypothesis that a working environment filled by organizational injustices can be a powerful stressor, since it requires constant adaptations of the individual to deal with such injustices. This work, of a theoretical nature, proposes a reflection regarding to the relation between the occupational stress and organization justice constructs, concluding from the literature that a working environment filled by injustices can be a potential stressor and points out the importance of investigating empirically this relation.
\end{abstract}

\footnotetext{
1 paulobenzoni@uol.com.br

2 jgomeservolino@hotmail.com

3 mikesiimons@gmail.com
} 
Keywords: Stress; Organizational Justice; Stressors.

\section{Résumé}

L'étude du stress professionnel, ses sources, sa dynamique de fonctionnement et ses conséquences ont été largement étudiées. Il existe un consensus selon lequel l'environnement de travail est une source puissante de stress professionnel. En prenant la construction de la justice organisationnelle, on fait l'hypothèse qu'un lieu de travail marqué par l'injustice organisationnelle peut être un puissant facteur de stress, puisqu'il nécessite des adaptations constantes de l'individu pour faire face à de telles injustices. Ce travail propose une réflexion sur la relation entre le stress professionnel et la justice organisationnelle, donc il est conclu de la littérature qu'un environnement de travail marqué par des injustices peut être un facteur de stress potentiel et souligne l'importance de l'étude empirique de cette relation.

Mots Clés: Stress; Justice Organis ationnelle; Facteurs de Stress.

\section{Resumen}

El estudio del estrés ocupacional, sus fuentes, dinámica de funcionamiento y consecuencias ha sido ampliamente estudiado. Existe un consenso de que el ambiente de trabajo consiste en una poderosa fuente del estrés ocupacional. Tomando el constructo de justicia organizacional, se considera la hipótesis de que un ambiente de trabajo marcado por injusticias organizacionales puede ser un poderoso estresor, ya que requiere adaptaciones constantes del individuo para enfrentar tales injusticias. Este trabajo, de n aturaleza teórica, propone una reflexión sobre la relación entre los constructos del estrés ocupacional y de la justicia organizacional, concluyendo a partir de la literatura que un ambiente laboral lleno de injusticias puede sí ser un estresor potencial y señala la importancia de investigar empíricamente esta relación.

Palabras claves: Estrés; Justicia Organizacional; Estresores.

\section{Introdução}

$\mathrm{Na}$ atualidade os espaços de trabalho têm sido marcados, cada vez mais, por ambientes competitivos, carregados de cobranças por produtividade e modelos de gestão que muitas vezes não se calcam na aplicação justa de procedimentos e normas organizacionais. Observa-se que em muitas organizações, que focam suas estratégias no atendimento das demandas de mercado, impera a ordem da produção sobre a ordem das relações interpessoais adequadas, gerando ambientes insalubres do ponto de vista da saúde mental e do estresse.

Para abordar a questão da aplicação "justa" de procedimentos e normas, pode-se ter como base o conceito de justiça organizacional, o qual se refere ao modo como as pessoas percebem a forma "padrão" existente na empresa e que determina o modo como o trabalho é organizado (Justiça de Procedimentos), os recursos são distribuídos (Justiça
Distributiva) e as relações interpessoais se estabelecem (Justiça Interpessoal). Fischer (2012), abordando as perspectivas atuais e os desafios nas pesquisas sobre justiça organizacional, indica uma relação entre esta, com a saúde e o bem-estar ocupacional. De modo a abordar os reflexos de ambientes de trabalho insalubres à saúde mental, toma-se o conceito de estresse, uma vez que este pode ser considerado como manifestação concreta dos problemas relativos à saúde e bem-estar no trabalho.

Integrando os dois conceitos, justiça organizacional e estresse, pode-se considerar então, que a justiça organizacional vem a ser um importante aliado para a compreensão da dinâmica do estresse ocupacional e propõe-se neste ensaio, uma reflexão, a partir da literatura atual, da relação existente entre ambos. 


\section{A resposta de estresse no ambiente de trabalho.}

$\mathrm{O}$ primeiro pesquisador a utilizar $\mathrm{o}$ termo estresse na literatura médica foi Hans Selye e de acordo com ele, em um sentido biológico, estresse é a interação entre perigo e defesa como o conceito físico de tensão ou pressão, ele representa a interação entre a força e a resistência oferecida (Selye, 1950). O estresse pode ser acarretado quando uma pessoa busca ajustar seus padrões habituais de comportamento, referente a qualquer demanda ambiental, social, biológica e/ou psicológica (Carr \& Umberson, 2013).

No que se refere ao estresse ocupacional, têm-se notado uma grande complexidade de estudos que buscam uma melhor compreensão deste fenômeno identificando causas e fatores que possam desencadeá-lo (Sousa, Silva \& GalvãoCoelho, 2015; Carr \& Umberson, 2013; Sadir, Bignotto \& Lipp, 2010; Sadir \& Lipp, 2009; Maissiat, Lautert, Dal Pai \& Tavares, 2015). Estes estudos apontam para o sentido de compreender se a consequência estressora ocorre devido ao ambiente social do trabalho, esforço físico ou relação entre ambos, e através dessa compreensão, criar estratégias que auxiliem a relação do trabalhador com o trabalho, bem como desenvolver tratamentos que ajudem a melhorar a saúde de quem já sofre desse mal.

Sousa, Silva e Galvão-Coelho (2015) relatam que quando o ambiente físico ou social está instável, respostas fisiológicas acontecem na tentativa de manutenção de homeostase para estabilizar o meio interno do indivíduo, apresentando uma sucessão de respostas integradas de enfrentamento. Essas respostas de enfrentamento auxiliam o indivíduo a se adaptar ao ambiente, contribuindo para sua sobrevivência.

Quando exigidas adaptações que vão além do limite do indivíduo, em condições favoráveis ou não, estas podem gerar como consequência o estresse (Sadir, Bignotto \& Lipp, 2010).

A literatura aponta como evidência, a relação do indivíduo e sua capacidade de enfrentamento, como mecanismos básicos da geração de estresse e, nesta linha, ao entrar no campo ocupacional, o modelo mais proeminente é o de demanda-controle proposto por Karasek nos anos de 1970. O autor propôs o modelo demanda-controle, que ao considerar os processos de produção do estresse ocupacional, destaca duas dimensões psicossociais no trabalho, a saber: a demanda psicológica proveniente do trabalho e o controle da tarefa. Desta forma, o modelo se propõe a diferenciar situações dentro do ambiente organizacional, compondo riscos à saúde do trabalhador (Karasek, 1979), o que se diferencia do modelo genérico de estresse, proposto por Selye, que, em linhas gerais, enfatizava a demanda e a capacidade de enfrentamento do indivíduo, omitindo sistematicamente o controle da tarefa da análise dos processos de produção do estresse (Araújo, Aquino, Menezes, Santos \& Aguiar, 2003),

Nesse escopo, o estresse emerge quando as exigências do trabalho excedem os recursos e as capacidades do sujeito, em um contexto organizacional no qual o trabalhador tem pouco apoio, autonomia e controle das suas atividades, de forma que é gerada uma série de respostas de cunho emocional, fisiológico e/ou comportamental, que limitam a ação da pessoa e facilitam a diminuição da saúde e do bem estar do sujeito (Laranjeira, 2009). Seguindo esta linha, faz-se interessante citar uma pesquisa, a qual aponta que a insegurança no trabalho e o baixo apoio social, estão positivamente associados com distúrbios mentais; e ao tomar o modelo demanda-controle como referência, foi constatado que aqueles que estiveram em situações de alta exigência e baixo controle da tarefa eram mais propensos a terem distúrbios mentais 
(Wang, Lesage, Schmitz \& Drapeau, 2008).

Há, sobretudo, o lado das diferenças interindividuais na resposta ao estresse. $\mathrm{O}$ organismo também pode funcionar em diversas condições de ajuste da homeostase, possibilitando uma melhor adaptação ao meio, o que é denominado de capacidade de alostase (Sousa, Silva \& Galvão-Coelho, 2015).

A energia liberada pelo organismo no conceito de alostase é conhecida como carga alostática e ajuda o mesmo atingir a estabilidade através da mudança. Quando em excesso, essa carga alostática pode desencadear patologias físicas ou mentais, as quais geram uma sobrecarga, incapacitando o indivíduo de ativar sistemas alostáticos diante de uma demanda, podendo desencadear um processo patológico (McEwen \& Wingfield, 2010).

Complementando a afirmação acima, segundo Souza, Silva e GalvãoCoelho (2015), o indivíduo que não possui boa habilidade em lidar com eventos desafiadores ou que os deixam vulneráveis, desestabilizam os sistemas alostáticos, ativando-os de forma inadequada, gerando uma falha nestes e desencadeando um desequilibrio em vários outros sistemas fisiológicos.

As tentativas de reajuste ao meio, quando não bem-sucedidas, ocasionam o estresse, fator preocupante devido às suas consequências para a saúde e qualidade de vida. Estes desajustes causam implicações também para a sociedade e para as empresas, pois prejudicam o desempenho e as atividades diárias do indivíduo, uma vez que diminui seu ritmo de trabalho (Sadir \& Lipp, 2009).

$\mathrm{O}$ ambiente de trabalho pode ser um grande desencadeador do estresse ocupacional, isto se dá quando o indivíduo avalia as demandas físicas e sociais do trabalho como demasiadas aos recursos que ele dispõe para gerenciá-las a partir de suas características individuais, este processo de avaliação define se o estresse irá ou não se instalar (Sadir, Bignotto \& Lipp, 2010).

O nível de estresse pode se diferir de acordo com o tipo de ocupação (Sadir, Bignotto \& Lipp, 2010). As características do trabalho e suas práticas, também são consideradas os pilares para entender o porquê em algumas organizações, os trabalhadores se encontram motivados, enquanto em outras, identifica-se uma insatisfação (Laranjeira, 2009), além disso, observa-se que as demandas sociais do trabalho podem ser geradoras de estresse. Tudo isso pode ser organizado no conceito de contexto de trabalho.

O contexto de trabalho diz respeito ao ambiente social e as condições laborais oferecidas pela organização, juntamente às relações profissionais operantes neste ambiente (Maissiat e colaboradores, 2015). Os mesmos autores ainda pontuam que as relações interpessoais na organização são muito importantes na saúde do trabalhador, pois estas determinam as vivências de prazer e sofrimento do sujeito em conjunto com as condições laborais que a organização oferece.

Laranjeira (2009), em estudo de revisão de literatura, aponta que o sofrimento gerado no estresse ocupacional gera respostas nos níveis físico, emocional e social, ocasionando prejuízos na vida do sujeito e, na mesma linha relativa ao contexto de trabalho, Benzoni, Barato, Marchezin e Inocente (2016), ao avaliarem a percepção deste contexto em trabalhadores afastados por motivo de doença osteomuscular, identificaram que a percepção nos fatores de condições de trabalho e relações socioprofissionais apresentaram diferenças altamente significativas entre trabalhadores sem estresse e com estresse. Quando com estresse, os trabalhadores percebem de forma mais negativa o contexto de trabalho, bem como aspectos relativos à carga de trabalho, além de identificarem mais deficiências nas relações socioprofissionais. 
As incoerências vividas pelo trabalhador em seu contexto laboral, como cobrança por resultados, tarefas repetitivas, ritmo de trabalho acelerado, sobrecarga de trabalho e o forte sistema hierárquico, podem favorecer a doença psíquica. Este sofrimento pode ser avaliado pelo esgotamento advindo do estresse acumulado e acaba gerando insegurança, sobrecarga, insatisfação ou medo (Maissiat e colaboradores, 2015).

\section{A Justiça organizacional e seus reflexos nos trabalhadores.}

Como colocado no início deste trabalho, a justiça organizacional, em linhas gerais, se refere ao modo como as pessoas percebem o quão justo é seu ambiente de trabalho nas questões relativas a procedimentos adotados, distribuição de recursos e relações interpessoais

A Justiça Organizacional trabalha em prol da relação saúde-doença no trabalho, investigando a distribuição, os procedimentos adotados na distribuição e interação entre empresa e trabalhador, buscando a igualdade e justiça no contexto de trabalho. Quando há desigualdade nesse ambiente, motiva-se a tensão e a busca de estratégias para alívio desta, fazendo com que o trabalhador apresente uma redução de desempenho, reivindicações e até mesmo absenteísmo (Assmar, Ferreira, Oliveira \& Souto, 2005). A busca da equidade, entendida como justiça, foca-se nas trocas existentes na organização, sendo elas econômicas ou sociais, que envolvem o trabalhador $\mathrm{e}$ a relação com seus superiores, fazendo com que se apresente, nessas relações, a ideia de justiça e merecimento (Assmar e colaboradores, 2005).

Um estudo que buscou investigar o impacto da justiça organizacional em atitudes e comportamentos específicos, tais como satisfação com o trabalho, comprometimento organizacional e intenção de rotatividade, concluiu que este conceito influencia diretamente nas atitudes e comportamentos citados. Um tratamento justo no que diz respeito à distribuição das recompensas, uso de procedimentos e o oferecimento de informações adequadas, detalhadas e precisas aos trabalhadores, apresenta um grande impacto na operação da organização (Rai, 2014).

Ainda nesta linha, Ibrahim e Perez (2014) também apontam que a justiça organizacional influencia diretamente na satisfação do trabalhador com o seu trabalho, a qual também produz impactos diretos em comportamentos relacionados ao comprometimento com o trabalho.

Os autores Leow \& Khong, 2015 e Moon, Hur, Ko, Kim \& Yoon, (2014), ao analisarem a relação da justiça organizacional com o comprometimento, pontuam que se esta está presente no local de trabalho, o trabalhador tende a ser mais comprometido com a organização, ou seja, sua percepção torna-se positiva e o indivíduo mostra-se mais satisfeito e comprometido com o seu trabalho e com a empresa.

Há uma correlação positiva entre comportamentos que visam contribuir para a produtividade da organização e a percepção de justiça organizacional de modo que, quanto maior for à percepção dos trabalhadores de que são tratados de maneira justa pela empresa e seus líderes, maior a tendência de se esforçarem mais para cooperar com a organização (Tziner \& Sharoni, 2014).

\section{O estresse e a (in)justiça organizacional.}

Se o estresse corresponde ao resultado da incapacidade do indivíduo lidar adequadamente com as demandas do ambiente e se a justiça organizacional corresponde à percepção de justiça nos procedimentos, distribuição e relacionamentos interpessoais, quando não houver justiça no ambiente 
organizacional, haverá maior probabilidade de se desenvolver estresse, já que um ambiente injusto, por natureza gera consideráveis demandas sobre os indivíduos que nele vivem.

Partindo desta hipótese, observamse alguns estudos que tendem a demonstrar esta relação entre o ambiente e as percepções do indivíduo.

Francis e Barling

confirmaram que as desigualdades apresentadas no ambiente de trabalho acarretam experiências estressantes, ocasionando sintomas de tensão ao indivíduo exposto neste contexto. Tziner e Sharoni (2014) apontam que a percepção de justiça no âmbito organizacional também se associa ao estresse, de modo que quanto maior a percepção de justiça por parte do trabalhador, menor são os indicadores de estresse apresentado pelo mesmo.

De modo empírico, Tziner e Sharoni (2014) propuseram e testaram um modelo onde demonstraram a relação entre justiça organizacional, comportamentos que contribuem para a organização, estresse e conflitos familiares. Os autores apontaram que quanto maior o nível de justiça organizacional percebido pelo trabalhador, maior a tendência dele apresentar comportamentos que contribuem para a produtividade da organização, o que leva a uma redução de seu estresse. Observaram, ainda, que o estresse é um elemento que influencia diretamente nos conflitos familiares e quando o nível de estresse diminui, há uma redução destes conflitos, sendo assim, a percepção de justiça organizacional afetaria os conflitos familiares.

Moon, Hur, Ko, Kim e Yoon (2014), apontam que elementos da justiça organizacional, a saber, justiça distributiva e a justiça interpessoal, em conjunto com o comprometimento afetivo (que sofre influência de tais elementos), exercem grande importância no desenvolvimento do senso de compaixão e cooperação entre os funcionários de uma organização, contribuindo para um melhor desempenho e envolvimento dos indivíduos com o trabalho.

Choi, Park, Yoon e Kim (2013), avaliando residentes de medicina, através de uma escala, com oito subcategorias, identificaram que a percepção de injustiça organizacional por parte do sujeito, foi a que teve a maior média de escore referente às fontes de estresse.

Outro estudo que objetivou identificar os fatores comuns que contribuíam para comportamentos contra produtivos de médicos e enfermeiros dos hospitais do Paquistão, mostrou que fatores como injustiça organizacional e quebra de contrato psicológico se associavam de maneira significativa a comportamentos inadequados dentro do trabalho por parte dos profissionais (Ahmed, Kiyani \& Hashmi, 2013).

Topa, Moriano e Morales (2013), estudaram os efeitos provocados pela percepção de injustiça no âmbito organizacional, por parte de terceiros, analisando a reação dos colegas de trabalho da vítima de situações consideradas injustas. Os resultados demonstraram que, mesmo não sendo diretamente afetados pela situação considerada incorreta ou injusta, colegas de trabalho podem experienciar emoções negativas intensas em decorrência de violações dos padrões normativos de moral ou comportamento social, gerando possíveis dificuldades em lidar com a demanda social da organização, sobre a qual se teria baixo ou nenhum controle.

\section{Considerações finais}

Pio Silva, Minette, Souza, Alvez Marçal e Petean Sanches (2013) dizem que o contexto de trabalho atual está gerando cada vez mais o estresse em seus trabalhadores. Pontuam que isto ocorre devido ao número de exigências impostas pelas empresas que obrigam o trabalhador 
a seguir as características da organização, intensificando seu trabalho e fazendo com que o mesmo não consiga se adequar à demanda exigida.

Por outro lado, Maissiat, Lautert, Dal Pai e Tavares (2015) afirmam que o trabalhador que tem seu trabalho reconhecido pela chefia, apresenta mais produtividade, alcance das metas propostas e atendimento às exigências do trabalho. $\mathrm{O}$ reconhecimento também apresenta papel positivo contra o "esgotamento" profissional.

Há, então, por um lado a configuração de um contexto de trabalho "injusto", gerador de estresse e de outro a configuração de um contexto "justo", gerador de motivação e comprometimento.

Retomando o modelo demandacontrole do estresse ocupacional, Theorell e Karasek (1990) consideram que quando se tem alta demanda e alto controle sobre o trabalho, o mesmo se mostra desafiador e motivador, ou seja, menos estressante do ponto de vista de adoecimento pelo trabalho. Tomando as colocações de Maissiat e colaboradores (2015), que retratam um contexto de trabalho "justo", tem-se, então algumas indicações da relação entre Justiça organizacional e estresse.

Aventou-se neste ensaio a hipótese de que, quando não houver justiça no ambiente organizacional, haverá maior probabilidade de se desenvolver estresse, já que um ambiente injusto por natureza acaba por gerar consideráveis demandas sobre os indivíduos que nele vivem. A partir das proposições teóricas aqui apresentadas, percebem-se indicadores de que a hipótese proposta é correta.

Pode, então, a justiça organizacional, quando percebida como injusta, constituir-se em fonte de estresse? A resposta parece ser "sim", e assim sendo observa-se a necessidade de estudos mais aprofundados que analisem empiricamente a validade da hipótese aqui levantada.
Compreender de forma clara esta relação entre justiça organizacional e Estresse Ocupacional poderá trazer inúmeros benefícios na condução de trabalhos de promoção da saúde neste contexto, prevenção do estresse e melhorias das condições salubres no trabalho, do ponto de vista da saúde mental.

\section{Referências}

Ahmed, W., Kiyani, A. A. \& Hashmi, S. H. (2016). The study on organizational cynicism, organizational injustice \& breach of psychological contract as the determinants of deviant work behavior. Actual Problems of Economics, 2 (140), 145-154. Disponível em: https://papers.ssrn.com/sol3/papers.cf m?abstract $\mathrm{id}=2743141$. Acesso em 04/01/2018.

Araújo, T. M., Aquino, E., Menezes, G., Santos, C. O. \& Aguiar, L. (2003). Aspectos psicossociais do trabalho e distúrbios psíquicos entre trabalhadoras de enfermagem. Revista de Saúde Pública, 37(4), 424-433. Disponível em: http://www.revistas.usp.br/rsp/article/ view/31611. Acesso em 28/12/2017.

Assmar, E. M. L., Ferreira, M. C. \& Souto, S. D. O. (2005). Justiça organizacional: uma revisão crítica da literatura. Psicologia: reflexão $e$ crítica, 18(3), 443-453. Disponível em:

http://www.scielo.br/pdf/\%0D/prc/v1 8n3/a19v18n3.pdf. Acesso em: 22/12/2017.

Benzoni, P. E, Barato, C. C., Marchezin, M. A. \& Inocente, M. M. (2016) Afastamento do trabalho e crise do capital: a incapacidade refletindo $\mathrm{o}$ 
contexto. Revista Ser Social, 18 (39), 540-561. Disponível em http://periodicos.unb.br/index.php/SE R_Social/article/view/21727/17530. Acesso em 20/09/2017.

Carr, D. \& Umberson, D. (2013). The social psychology of stress, health, and coping. In Handbook of social psychology. Netherlands: Springer. Disponível em: https:/link.springer.com/chapter/10.1 007/978-94-007-6772-0_16. Acesso em 05/01/2018.

Choi, S. M., Park, Y. S., Yoo, J. H. \& Kim, G. Y. (2013). Occupational stress and physical symptoms among family medicine residents. Korean journal of family medicine, 34(1), 4957. Disponível em: https:/synapse.koreamed.org/DOIx.p hp?id=10.4082/kjfm.2013.34.1.49.

Acesso em 05/01/2018.

Fischer, R. (2012). Organizational justice research: present perspectives and challenges. Revista Psicologia Organizações e Trabalho, 12(1), 97112. Disponível em: http://pepsic.bvsalud.org/scielo.php?p $\mathrm{id=S198466572012000100009 \& \text {scrip }}$ $\mathrm{t}=$ sci_abstract\&tlng=en. Acesso em 04/01/2018.

Francis, L. \& Barling, J. (2005). Organizational injustice and psychological strain. Canadian Journal of Behavioural Science/Revue canadienne des sciences du comportement, 37(4), 250-261. Disponível em: http://psycnet.apa.org/record/200514046-002. Acesso em 15/12/2017.

Ibrahim, M. E. \& Perez, A. O. (2014). Effects of organizational justice, employee satisfaction, and gender on employees' commitment: evidence from the UAE. International Journal of Business and Management, 9(2),
45-59. Disponível em: http://www.ccsenet.org/journal/index. php/ijbm/article/view/30434. Acesso em 05/01/2018.

Karasek Jr, R. A. (1979). Job demands, job decision latitude, and mental strain: Implications for job redesign. Administrative science quarterly, 24 (2) 285-308. Disponível em: http://www.jstor.org/stable/2392498. Acesso em 15/12/2017.

Laranjeira, C. A. (2009). O contexto organizacional e a experiência de estresse: uma perspectiva integrativa. Rev Salud Publica, 11(1), 123-33. Disponível em: https://scielosp.org/pdf/rsap/v11n1/v1 1n1a13.pdf. Acesso em 15/12/2017.

Leow, K. L. \& Khong, K. W. (2015). Organizational commitment: The study of organizational justice and leader-member exchange (LMX) among auditors in Malaysia. International journal of business and information, 4(2), 161-198. Disponível em: http://ijbi.org/ijbi/article/view/42.

Acesso em 05/01/2018.

Maissiat, G. D. S., Lautert, L., Dal Pai, D. \& Tavares, J. P. (2015). Contexto de trabalho, prazer e sofrimento na atenção básica em saúde. Revista gaúcha de enfermagem (Porto Alegre), 36 (2), 42-49. Disponível em:

https://www.lume.ufrgs.br/handle/101 83/130175. Acesso em 04/01/2018.

McEwen, B. S. \& Wingfield, J. C. (2010). What's in a name? Integrating homeostasis, allostasis and stress. Hormones and behavior, 57(2), 1-16. Disponível em: https://www.ncbi.nlm.nih.gov/pmc/art icles/PMC2815096. Acesso em 10/01/2018. 
Moon, T. W., Hur, W. M., Ko, S. H., Kim, J. W. \& Yoon, S. W. (2014). Bridging corporate social responsibility and compassion at work: Relations to organizational justice and affective organizational commitment. Career Development International, 19(1), 4972. Disponível em: http://www.emeraldinsight.com/doi/f ull/10.1108/CDI-05-2013-0060. Acesso em 05/01/2018.

Pio Silva, E., Minette, L. J., Souza, A. P., Alves Marçal, M. \& Petean Sanches, A. L. (2013). Fatores Organizacionais e psicossociais associados ao risco de LER/DORT em operadores de máquinas de colheita florestal. Revista Árvore, 37(5), 889895. Disponível em: http://www.redalyc.org/htm//488/488 29247011/. Acesso em 14/12/2017.

Rai, G. S. (2013). Impact of organizational justice on satisfaction, commitment and turnover intention: Can fair treatment by organizations make a difference in their workers' attitudes and behaviors? Journal of Human Sciences, 10(2), 260-284. Disponível em: https://jhumansciences.com/ojs/index.php/ijh s/article/view/2634. Acesso em 04/01/2018.

Sadir, M. A. \& Lipp, M. E. N. (2009). As fontes de stress no trabalho. Revista de Psicologia da IMED, 1(1), 114126. Disponível em: https $/ /$ seer.imed.edu.br/index.php/rev istapsico/article/view/16. Acesso em 15/12/2017.

Sadir, M. A., Bignotto, M. M. \& Lipp, M. E. N. (2010). Stress e qualidade de vida: influência de algumas variáveis pessoais. Paideia, 20(45), 73-81. Disponível em: http://www.redalyc.org/html/3054/30 5423775010. Acesso em 15/12/2017.
Selye, H. (1950). The physiology and pathology of exposure to stress. Montreal: Acta Medical Publishers.

Sousa, M. B. C. D., Silva, H. P. A. \& Galvão-Coelho, N. L. (2015). Resposta ao estresse: I. Homeostase e teoria da alostase. Estudos de Psicologia (Natal), 20(1), 2-11. Disponível em: http://www.scielo.br/scielo.php?pid= S1413294X2015000100002\&script $=\mathrm{S}$ ci_abstract\&thng=es. Acesso em 16/12/2017.

Theorell, T. \& Karasek, R. A. (1996). Current issues relating to psychosocial job strain and cardiovascular disease research. Journal of occupational health psychology, 1(1), 9-26. Disponível em:

http://psycnet.apa.org/record/199604477-002. Acesso em 13/12/2017.

Topa, G., Moriano, J. A. \& Morales, J. F. (2013). Organizational injustice: third parties' reactions to mistreatment of employee. Psicothema, 25(2), 214221.Disponível em: http://www.redalyc.org/html/727/727 26347002/. Acesso em 04/01/2018.

Tziner, A., \& Sharoni, G. (2014). Organizational citizenship behavior, organizational justice, job stress, and workfamily conflict: Examination of their interrelationships with respondents from a non-Western culture. Revista de Psicología del Trabajo y de las Organizaciones, 30(1), 35-42. Disponível em: http://www.sciencedirect.com/science /article/pii/S1576596214700295. Acesso em 04/01/2018.

Wang, J., Lesage, A., Schmitz, N. \& Drapeau, A. (2008). The relationship between work stress and mental disorders in men and women: findings from a population-based 
study. Journal of Epidemiology \&

Community Health, 62(1), 42-47.

Disponível em:

http://jech.bmi.com/content/62/1/42.s

hort. Acesso em 04/01/2018. 